\title{
Haplotype analysis of sucrose synthase gene family in three Saccharum species
}

\author{
Jisen Zhang ${ }^{1,2}$, Jie Arro ${ }^{2}$, Youqiang Chen ${ }^{1}$ and Ray Ming ${ }^{2 *}$
}

\begin{abstract}
Background: Sugarcane is an economically important crop contributing about $80 \%$ and $40 \%$ to the world sugar and ethanol production, respectively. The complicated genetics consequential to its complex polyploid genome, however, have impeded efforts to improve sugar yield and related important agronomic traits. Modern sugarcane cultivars are complex hybrids derived mainly from crosses among its progenitor species, S. officinarum and S. spontanuem, and to a lesser degree, S. robustom. Atypical of higher plants, sugarcane stores its photoassimilates as sucrose rather than as starch in its parenchymous stalk cells. In the sugar biosynthesis pathway, sucrose synthase (SuSy, UDP-glucose: D-fructose 2-a-D-glucosyltransferase, EC 2.4.1.13) is a key enzyme in the regulation of sucrose accumulation and partitioning by catalyzing the reversible conversion of sucrose and UDP into UDP-glucose and fructose. However, little is known about the sugarcane SuSy gene family members and hence no definitive studies have been reported regarding allelic diversity of SuSy gene families in Saccharum species.
\end{abstract}

Results: We identified and characterized a total of five sucrose synthase genes in the three sugarcane progenitor species through gene annotation and PCR haplotype analysis by analyzing 70 to 119 PCR fragments amplified from intron-containing target regions. We detected all but one (i.e. ScSuSy5) of ScSuSy transcripts in five tissue types of three Saccharum species. The average SNP frequency was one SNP per 108 bp, 81 bp, and 72 bp in S. officinarum, S. robustom, and S. spontanuem respectively. The average shared SNP is 15 between S. officinarum and S. robustom, 7 between S. officinarum and S. spontanuem, and 11 between S. robustom and S. spontanuem. We identified 27, 35, and 32 haplotypes from the five ScSuSy genes in S. officinarum, S. robustom, and S. spontanuem respectively. Also, 12, 11, and 9 protein sequences were translated from the haplotypes in S. officinarum, S. robustom, S. spontanuem, respectively. Phylogenetic analysis showed three separate clusters composed of SbSuSy 1 and SbSuSy2, SbSuSyz and SbSuSy5, and SbSuSy4.

Conclusions: The five members of the SuSy gene family evolved before the divergence of the genera in the tribe Andropogoneae at least 12 MYA. Each ScSuSy gene showed at least one non-synonymous substitution in SNP haplotypes. The SNP frequency is the lowest in S. officinarum, intermediate in S. robustum, and the highest in S. spontaneum, which may reflect the timing of the two rounds of whole genome duplication in these octoploids. The higher rate of shared SNP frequency between S. officinarum and S. robustum than between S. officinarum and in S. spontaneum confirmed that the speciation event separating S. officinarum and S. robustum occurred after their common ancestor diverged from S. spontaneum. The SNP and haplotype frequencies in three Saccharum species provide fundamental information for designing strategies to sequence these autopolyploid genomes.

Keywords: Sucrose synthase, Haplotype, Single nucleotide polymorphisms, Saccharum officinarum, Saccharum spontaneum, Saccharum robustum

\footnotetext{
* Correspondence: rming@life.illinois.edu

${ }^{2}$ Department of Plant Biology, University of Illinois at Urbana-Champaign,

Urbana, IL 61801, USA

Full list of author information is available at the end of the article
}

\section{Biomed Central}

(c) 2013 Zhang et al.; licensee BioMed Central Ltd. This is an Open Access article distributed under the terms of the Creative Commons Attribution License (http://creativecommons.org/licenses/by/2.0), which permits unrestricted use, distribution, and reproduction in any medium, provided the original work is properly cited. 


\section{Background}

Sugarcane (Saccharum spp.) is an agronomically important grass that contributes about $80 \%$ of the world sugar production (FAOSTAT, 2010) and, more recently, has become a major biofuel feedstock, contributing about $40 \%$ of ethanol production worldwide [1]. Consequently, sugarcane breeding efforts is now largely geared towards improvement in both sugar and biomass yield.

Although considerable improvement has been made in sugar yield in the past decades, sugarcane is substantially lagging behind most crops in maximizing gains through molecular breeding. Most of the basic molecular genetic analyses remains unresolved in sugarcane due to unique challenges and complications brought about by a genome with an extreme autoploidy level that can range from octoploidy $(x=8)$ to dodecaploidy $(x=12)$. The saccharum complex have no known diploid member species but are all polyploids. S. officinarum's chromosome number is constant at $2 \mathrm{n}=80$ while that for $S$. spontaneum and $S$. robustum ranges from $2 n=36-128$ and $2 n=60-160$, respectively [2]. S. spontaneum have a basic chromosome number $\mathrm{x}=8$ while both $S$. officinarum and $S$. robustum would have $x=10$ [3]. Modern sugarcane cultivars are complex autopolyploid and aneuploids of interspecific hybrids derived from $S$. officinarum, S. spontaneum and $S$. robustum. About $80-90 \%$ of modern day cultivars' chromosomes are derived from S. officinarum and the remaining $10-20 \%$ are derived from S. spontaneum, and inter-specific recombination [4-6]. Hybrid cultivars' high sugar content trait is contributed by $S$. officinarum, while the stress tolerance and pest and disease resistance is attributed by $S$. spontaneum. More recently, another well-known vigorous growing wild species, $S$. robustum, is being tapped in some sugarcane breeding programs for enhanced biomass yield.

Due to its high degree of polyploidy and heterozygosity, sequencing the sugarcane genome using the current short-read sequencing technology remains a formidable challenge. For the most part, expressed sequence tags (EST) resources have been the sole resource for sugarcane gene and gene family discovery $[7,8]$. The recent sequencing and annotation of sorghum bicolor's genome, the closest diploid relative of sugarcane in the Andropogonae tribe, has served as an indispensable resource for sugarcane genomic studies [9]. Sorghum's genome size of about $730 \mathrm{Mb}$ [9] is roughly similar to the monoploid genome size of $S$. spontaneum of approximately $843 \mathrm{Mb}[3]$. The high degree of synthenic collinearity that has been reported by linkage mapping [10-12] and sequence comparison of selected sugarcane bacterial artificial chromosomes (BACs) $[9,13,14]$ have provided some resolution on the complex genetics and inheritance of sugarcane.

Understandably, because sugarcane is grown largely for its sugar and its sugar-derived products like ethanol, gene families related to sucrose metabolism are of paramount importance and are the subject of rigorous molecular genetics interest. Sucrose synthase (SuSy, UDP-glucose: Dsfructose 2-a-D-glucosyltransferase, EC 2.4.1.13) is a major enzyme involved in sucrose metabolism [15-18] and partitioning [19] and is particularly important due to the unique ability of sugarcane to store its photoassimilates in the form of sucrose in its stalks [19-21]. A small multigene family has been found to encode several SuSy isoforms in many plant species including maize [22,23], rice [24], Arabidopsis [25] and some other model plant organisms $[26,27]$. However, aside from an expression analysis of a sugarcane SuSy cDNA [21] and a survey in sugarcane EST library, which revealed four SuSy clones highly homologous to SuSy isoform I [28], little is known about the variation in haplotypes of genes within and among Saccaharum species. Due to the complexity of the genome and the lack of whole genome sequence of sugarcane, studies dealing with haplotype analysis of gene families have received little attention.

Previously, the haplotypes of sucrose phosphate synthase III gene were surveyed to examine the association between SNP frequency and sucrose content in sugarcane and its progeny [29]. Haplotype sequences were analyzed for a target genomic region containing a brown rust resistance gene Brul in seven BACs from hybrid cultivar R570, and four, two, and two BACs were classified as S. officinarum, S. spontaneum, and recombinant haplotypes, respectively [30]. These are the only two studies for sugarcane haplotype sequences, and both used commercial hybrid cultivars as experiment materials, Q165 in the first study and R570 in the second. In order to understand the intra- and inter-species allelic variation of such an important gene like SuSy, we surveyed the single nucleotide polymorphisms (SNPs) and haplotypes variation in three founding species for modern sugarcane, S. officinarum $(x=10), S$. spontanuem $(x=8)$, and $S$. robustom $(x=8)$. We characterized the SuSy gene family members, its evolutionary origin, and the haplotype classes in the three Saccharum species known to be the progenitor to modern sugarcane.

\section{Results}

\section{Identification of five SuSy genes in sorghum}

We used the six well-annotated sucrose synthase genes in Arabidopsis thaliana (TAIR database) to find the corresponding homologous sucrose synthase gene family members in Sorghum bicolor (referred from here on as SbSusy). Of the five homologous SbSuSy genes identified, two were not annotated in the sorghum gene database (Phytozome database version 9, ftp://ftp.jgi-psf.org/pub/ compgen/phytozome/v9.0/Sbicolor_v1.4/). The sequences and location of these five annotated SbSuSy genes are listed in Table 1. 
Table 1 Sequence similarity of SuSy gene fragments between Saccharum and Sorghum bicolor

\begin{tabular}{|c|c|c|c|c|c|}
\hline \multicolumn{3}{|c|}{ Sorghum } & \multicolumn{2}{|c|}{ Sugarcane } & \multirow{2}{*}{$\begin{array}{c}\text { DNA } \\
\text { sequences } \\
\text { identity** }\end{array}$} \\
\hline Gene name & Chromosome position & Protein size & Gene name & & \\
\hline \multirow[t]{3}{*}{ SbSusy 1 Sb01g033060 } & Chr. 1 & 816 & ScSusy 1 & S. officinarum & $94 \%$ \\
\hline & & & & S. robustum & $94 \%$ \\
\hline & & & & S. spontaneum & $94 \%$ \\
\hline \multirow[t]{3}{*}{ *SbSusy 2} & Chr. 10 & 837 & ScSusy 2 & S. officinarum & $94 \%$ \\
\hline & & & & S. robustum & $94 \%$ \\
\hline & & & & S. spontaneum & $94 \%$ \\
\hline \multirow[t]{3}{*}{ SbSusy 3 Sb04g038410 } & Chr. 4 & 838 & ScSusy 3 & S. officinarum & $96 \%$ \\
\hline & & & & S. robustum & $96 \%$ \\
\hline & & & & S. spontaneum & $96 \%$ \\
\hline \multirow[t]{3}{*}{ SbSusy 4 Sb01g035890 } & Chr. 1 & 809 & ScSusy 4 & S. officinarum & $95 \%$ \\
\hline & & & & S. robustum & $96 \%$ \\
\hline & & & & S. spontaneum & $95 \%$ \\
\hline \multirow[t]{3}{*}{ *SbSusy 5} & Chr. 10 & 892 & ScSusy 5 & S. officinarum & $95 \%$ \\
\hline & & & & S. robustum & $95 \%$ \\
\hline & & & & S. spontaneum & $95 \%$ \\
\hline
\end{tabular}

Notes: *SbSusy2 and SbSusy5 were not annotated in the Sorghum genome.

** The sequence identity id between sorghum and sugarcane orthologous genes.

SuSy gene family is comprised of five genes in sugarcane The SuSy genes in both Arabidopsis thaliana and Sorghum bicolor were subsequently used to annotate and predict the corresponding SuSy gene family members in sugarcane (referred from here on as ScSusy) from the available sugarcane EST database (i.e. sugarcane assembled sequence (SAS)) and RNA-seq data generated in our laboratory. Each of the predicted sugarcane ScSuSy genes was verified by sequencing the PCR product amplified from genomic DNA samples of the three accessions: LAPurple (S. officinarum), SES208 (S. spontaneum), and Molokai6081 (S. robustum) (Table 1). The amplified PCR fragments showed an average of $95 \%$ sequence similarity to sorghum SuSy genes. ScSuSy1 and ScSuSy2 showed lower sequence similarity with their sorghum counterparts than the other three ScSuSy genes (Table 1).

RT-PCR was performed to detect the expression patterns of these five SuSy genes for each species in five tissues: leaf roll, mature leaves, the 3rd, 9th, and 15th internode. All except one (i.e. ScSuSy5) was consistently detected in all five tissue of each sugarcane species (Figure 1).

We assembled the short-read cDNA sequences for each of the five $\mathrm{ScSuSy}$ genes derived from RNA-seq analyses of LA Purple leaf tissue (R. Ming, unpublished data). The amino acid sequences were deduced from open reading frames (ORFs) and homology-based analyses (Table 2). The predicted molecular weights of the five polypeptides range from 91.71 to $98.79 \mathrm{kDa}$ while the predicted isoelectric point of the polypeptides range from $5.82(\mathrm{ScSuSy} 2)$ to 8.26 (ScSuSy3). We found that the predicted amino acid

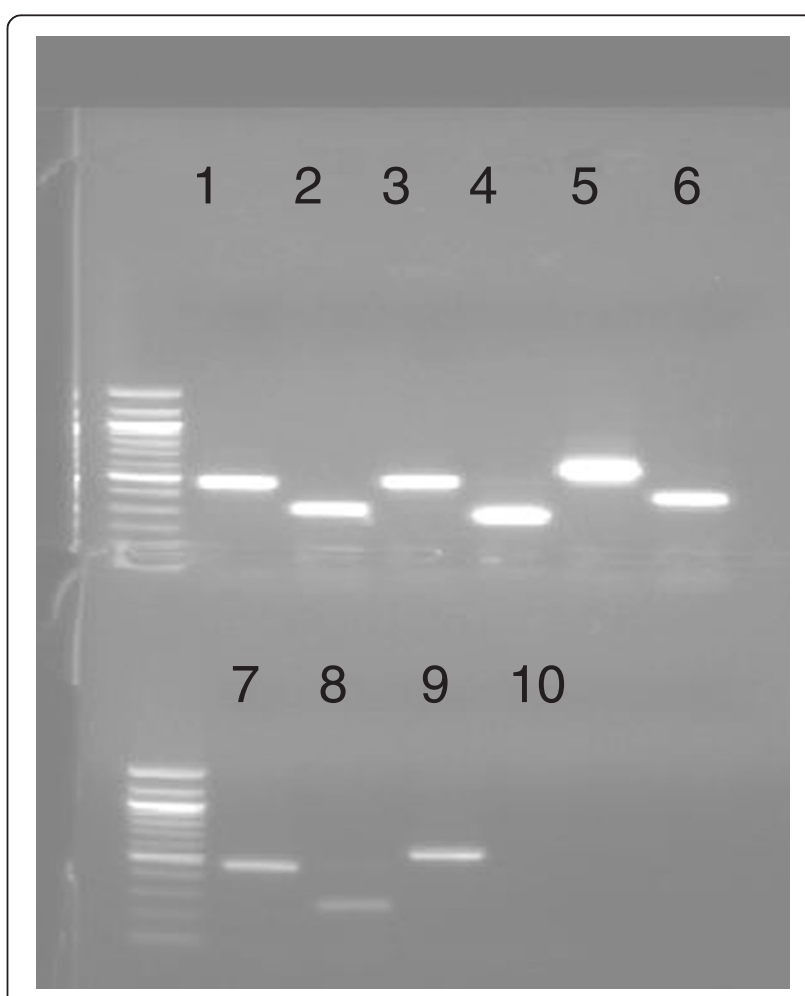

Figure 1 Results of RT-PCR and genomic PCR amplification of the $\mathbf{5}$ SuSy genes in S. $\boldsymbol{o}$. (S. officinarum). Lanes 1: Genomic PCR of SuSy1; 2:RT-PCR of SuSy1; 3: Genomic PCR of SuSy2; 4: RT-PCR of SuSy2; 5: Genomic PCR of SuSy3 DNA; 6: RT-PCR of SuSy3; 7: Genomic PCR of SuSy4; 8 RT-PCR of SuSy4 ; 9: Genomic PCR of SuSy5; 10:RT-PCR of SuSy5. 
Table 2 Pairwise similarity index between the five predicted ScSuSy amino acid sequences in sugarcane

\begin{tabular}{cccccc}
\hline & \multicolumn{5}{c}{ Similarity } \\
\hline Gene & ScSuSy1 & ScSuSy2 & ScSuSy3 & ScSuSy4 & ScSuSy5 \\
Protein size & 816 & 802 & 824 & 806 & 866 \\
molecular mass (KDa) & 92.96 & 91.71 & 93.59 & 91.95 & 98.79 \\
Isoelectric point (pl) & 6.03 & 5.82 & 8.26 & 6.29 & 6.56 \\
ScSuSy1 & - & - & - & - & - \\
ScSuSy2 & 0.80 & - & - & - & - \\
ScSuSy3 & 0.51 & 0.53 & - & - & - \\
ScSuSy4 & 0.70 & 0.71 & 0.54 & - & - \\
ScSuSy5 & 0.54 & 0.54 & 0.71 & 0.55 & - \\
\hline
\end{tabular}

sequences between ScSuSy1, ScSuSy2 and ScSuSy4 share a consistently higher pairwise sequence similarity $(70-80 \%)$ in contrast to ScSuSy3 and ScSuSy5 (< 70\%).

\section{Phylogenetic analysis of SuSy orthologous genes in sugarcane and sorghum}

To see the sequence similarity and evolutionary relationship among the SbSuSy gene family members in sorghum, an unrooted phylogenetic tree was generated using the full length protein sequences of the SbSuSy genes. The phylogenetic tree constructed by the neighbor-joining method formed two well-defined clusters. One cluster contained SbSuSy3 and SbSuSy5, and the other contained SbSuSy1, SbSuSy2 and SbSuSy4 (Figure 2).

For comparison, an unrooted phylogenetic tree was likewise constructed for assessing the evolutionary relationship of SuSy genes of sorghum and several well-annotated plant and bacterial genomes. Twenty-eight protein sequences from dicots, 26 sequences from monocots, and 4

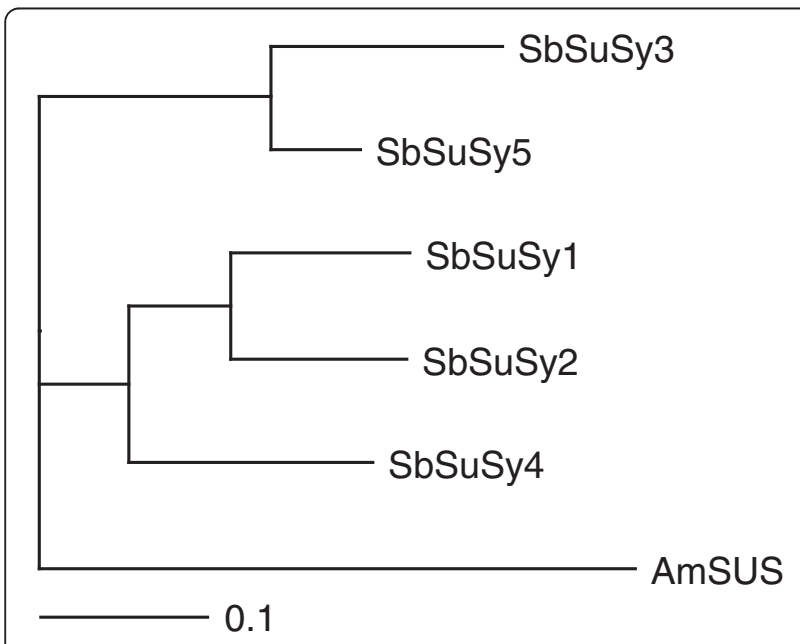

Figure 2 Phylogenetic relationships between sorghum sbSuSy with Anabaena ASuSy (a filamentous cyanobacteria) as an outgroup. bacteria sequences were used for constructing the unrooted phylogenetic tree (Additional file 1). All of the bacterial SuSy genes clustered into the same group (outgroup), distinctly branching away from the plant SuSy gene cluster. The SuSy genes of angiosperms could be subdivided into three distinct subgroups, arbitrarily designated as Class I, II and III (Figure 3). SbSuSy1 and SbSuSy2, SbSuSy3 and SbSuSy5, and SbSuSy4 were distributed in Class I, II and III, respectively (Figure 3). Interestingly, Class I and II seem to reflect the boundary between monocots and dicots.

Identification of SNPs in the five ScSuSy genes within and among Saccharum Species

To compare sequence variation and identify single nucleotide polymorphism (SNP) among the five annotated ScSuSy genes within and among Saccharum species, we designed PCR primers that will amplify a $500 \mathrm{bp}$ region that includes both an exonic and intronic sequences. To reduce the potential confounding issue of intergenomic recombination, SNPs were only reported if found in at least three sequences. To ensure sufficient sequencing depth in octoploids, 70 to 119 amplified fragments were cloned and sequenced per gene per species.

In ScSuSy1, four, seven, and eleven single nucleotide polymorphisms (SNPs) were detected within the $489 \mathrm{bp}$ region in S. officinarum (LA Purple), S. robustum (Molokai6081), and S. spontaneum (SES208), respectively. Of the total 22 SNPs, 19 were found within introns. One of these intronic SNPs is consistently present in all three species. In ScSuSy2, four, three, and six SNPs were identified within the 484 bp region in the three respective species. In this case, however, none of the SNPs within ScSuSy2 are shared in all three species. Of the combined 13 SNPs from the three species, only 5 occurred in introns. ScSuSy3 had seven SNPs in the 569 bp region in each of the three species, and four of these SNPs were identical in all the three species. ScSuSy4 had seven, nine, and nine SNPs from the above three species in the 470 bp region, respectively. Only one SNP is shared by the three species. In ScSuSy5, two, six, and three SNPs were found within a $577 \mathrm{bp}$ region of the three respective species, but none was common among the three species(Table 3, Additional files 2 and 3).

SNPs identified from five ScSuSy gene fragments were combined to estimate the SNP frequency within the genome of each species. The highest SNP frequency, at one SNP per $72 \mathrm{bp}$, is in the genome of the wild species $S$. spontanuem; the lowest SNP frequency, at one SNP per $108 \mathrm{bp}$, is in the genome of the domesticated high sugar content species S. officinarum. The SNP frequency in the genome of the wild species $S$. robustum is about one SNP per $81 \mathrm{bp}$, closer to that of the S. spontaneum (Table 3). However, pairwise DNA sequence comparison 


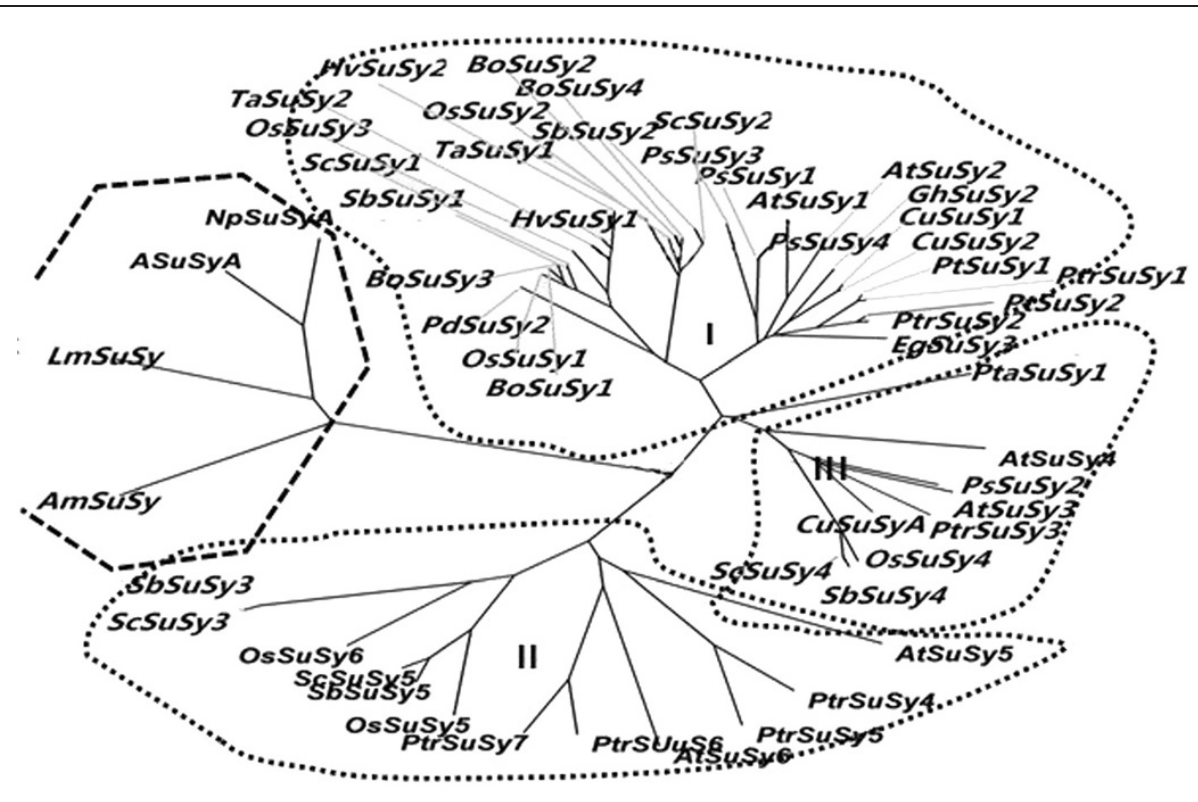

0.1

Figure 3 An unrooted phylogenetic tree derived from SuSy protein sequences of sorghum, sugarcane and the other plants (refer to Additional file 1).

Table 3 SNP counts per base pair in the five ScSuSy fragments within and between the three Saccharum species

\begin{tabular}{|c|c|c|c|c|c|c|c|}
\hline Saccharum species SNP & ScSuSy1 & ScSuSy2 & ScSuSy3 & ScSuSy 4 & ScSuSy5 & total & bp/SNP \\
\hline S. $O$. & $4 / 489$ & $4 / 484$ & $7 / 569$ & $7 / 470$ & $2 / 577$ & $24 / 2589$ & 108 \\
\hline S. $r$. & $7 / 489$ & $3 / 484$ & $7 / 569$ & $9 / 470$ & $6 / 577$ & $32 / 2589$ & 81 \\
\hline S. s. & $11 / 489$ & $6 / 484$ & $7 / 569$ & $9 / 470$ & $3 / 577$ & $38 / 2589$ & 72 \\
\hline \multicolumn{8}{|c|}{ Total SNPs count between the Saccharum species } \\
\hline S. o. + S.r. & $10 / 489$ & $5 / 484$ & $8 / 569$ & $12 / 470$ & $7 / 577$ & $42 / 2589$ & 62 \\
\hline S. o. + S. S. & $14 / 489$ & $9 / 484$ & $10 / 569$ & $15 / 470$ & $5 / 577$ & $53 / 2589$ & 49 \\
\hline S. $r+S . s$. & $16 / 489$ & $9 / 484$ & $10 / 569$ & $15 / 470$ & $8 / 577$ & $58 / 2589$ & 45 \\
\hline S. S. +S. o. + S.r. & $20 / 489$ & $10 / 484$ & $11 / 569$ & $18 / 470$ & 9/577 & $51 / 2589$ & 38 \\
\hline \multicolumn{8}{|c|}{ Identical SNPs count between the Saccharum species } \\
\hline S. o. IS.r. & $2 / 489$ & $2 / 484$ & $6 / 569$ & $4 / 470$ & $1 / 577$ & $15 / 2589$ & 172 \\
\hline S. o.IS. S. & $1 / 489$ & $1 / 484$ & $4 / 569$ & $1 / 470$ & 0 & $7 / 2589$ & 370 \\
\hline S. s. IS.r. & $3 / 489$ & 0 & $4 / 569$ & $3 / 470$ & $1 / 577$ & $11 / 2589$ & 238 \\
\hline S. S. \S. o. \S.r. & $1 / 489$ & 0 & $4 / 569$ & $1 / 470$ & 0 & $6 / 2589$ & 435 \\
\hline
\end{tabular}

Note: S. o. : S. officinarum, S. r: S. robustum, S. s:S. spontaneum. 
between species revealed higher SNP frequency differences. The highest SNP frequency is between the two wild species S. robustum and S. spontaneum at one SNP per 38 bp. The lowest is between the domesticated species $S$. officinarum and the wild species $S$. robustum, which share the same basic chromosome number (Table 3).

Haplotype analysis of ScSuSy Genes of Saccharum Species The unique combinations of SNPs in each sequenced fragment within each species were used to define haplotypes. The number of haplotypes within each gene fragments ranged from three to eight (Table 4). In ScSuSy3, each of the three species reached the maximum 8 haplotypes while the other four ScSuSy genes have varying numbers of haplotypes in the three species. When the combined haplotypes from all five gene fragments were estimated for each species, we identified 27, 35, and 32 haplotypes in LA Purple (S. officinarum), Molokai6081 (S. robustum), and SES208 (S. spontaneum), respectively.

We also noted consensus haplotypes among the Saccharum ScSuSys genes species (Table 5). The majority of consensus haplotypes are expected to come from multiple homologous chromosomes, which are assumed to be the original haplotypes from the Saccharum species. The frequencies of consensus haplotypes are significantly higher than the other haplotypes (Additional file 4). In the total 75 haplotypes of the $5 \mathrm{ScSuSy}$ genes from the three species, 16 of them are consensus haplotypes with a frequency of $52.1 \%$ of the genes fragment, which is common between at least two of the species. Obviously, there are more gene alleles from the consensus haplotypes than from the other haplotypes. Of the consensus haplotypes, 3 were present in the all three species, 1 in ScSuSy2 and 2 in ScSuSy3. These can be assumed to have existed prior to the divergence of Saccharum species due to it is low possibility for common haplotypes were from occasional mutational event. Pairwise comparison between $S$. officinarum and $S$. spontaneum, $S$. spontaneum and $S$. robustum, and $S$. officinarum and S. robustum revealed 4, 6 and 15 haplotypes that are similar to each other. This provides

Table 4 Estimated number of haplotypes of SuSy genes in three Saccharum species

\begin{tabular}{lcccccc}
\hline \multicolumn{7}{c}{ Haplotypes count } \\
\hline Species & SuSy1 & SuSy2 & SuSy3 & SuSy4 & SuSy5 & Summary \\
\hline S. $0(2 \mathrm{n}=80)$ & 4 & 6 & 8 & 6 & 3 & 27 \\
S. r. $(2 \mathrm{n}=80)$ & 6 & 5 & 8 & 8 & 8 & 35 \\
S. s. $(2 \mathrm{n}=64)$ & 8 & 7 & 8 & 5 & 4 & 32 \\
Total* & 15 & 15 & 16 & 17 & 11 & $74^{*}$
\end{tabular}

Notes:

* Due to the consensus haplotypes among the species, the total haplotypes numbers are different from the sum of each gene.

Note: S. o.: S. officinarum, S. r: S. robustum, S. s: S. spontaneum
Table 5 Number of deduced amino acid sequences for haplotypes fragments for each SuSy genes in the Saccharum species

\begin{tabular}{lcccccc}
\hline & ScSuSy1 & ScSuSy2 & ScSuSy3 & ScSuSy4 & ScSuSy5 & Total \\
\hline S. o & 1 & 1 & 4 & 3 & 3 & 12 \\
S. r. & 1 & 2 & 3 & 4 & 1 & 11 \\
S. s & 1 & 2 & 3 & 2 & 1 & 9 \\
total & 1 & 3 & 5 & 7 & 3 & 19
\end{tabular}

Notes: *The total deduced amino acid numbers are different from the sum of each gene because we presented the consensus deduced amino acid sequences among the species.

Notes: S. o : S. officinarum, S. r: S. robustum, S. s: S. spontaneum.

additional evidence in support of the contention that the ScSuSy families between S. officinarum and S. robustum is closer than that of the other two combinations.

The corresponding amino acid sequences of each haplotype were predicted by BlastX and aligned with ClustalW (Additional file 5). Except for ScSuSy1, which had no non-synonymous haplotypes, there were $3,5,5$ and 3 amino acids sequences predicted for ScSuSy2, ScSuSy3, ScSuSy4 and ScSuSy5, respectively. This highly suggests that multiple haplotypes results in the variation of amino acid sequence. It should be noted that the number of deduced protein sequences of haplotypes range from 1 to 7 for any of the ScSuSy genes of the three species, which is less than the haplotype number (Table 5). Obviously, the different haplotypes may still result in same protein sequence.

Using the information from the identification of the intron-exon boundaries for each scSuSY haplotype for each for each saccharum population-species, we calculated the pairwise synonymous substitutions (dS) and nonsynonymous $(\mathrm{dN})$ substitutions as described earlier [14]. Substitutions per synonymous site, or Ks values for each gene pairs between species were calculated using NeiGojobori method implemented in PAML [31]. Gene pairs giving unusually large Ks values, either because the sampled region were dissimilar or failed during the PAML calculation were discarded in the summary statistics. There were 19 pairs that meet this criteria, of which ten had $\mathrm{Ka}>=\mathrm{Ks}$. These values are affixed as Additional file 6 .

\section{Discussion}

Sugarcane was domesticated about 10,000 years ago and intensive artificial selection occurred only 100 years ago mostly on interspecific hybrids, not pure S. officinarum clones. Domestication, which was mainly on sugar content, might account for a small fraction of the reduced diversity in S. officinarum genome, but would not explain the lower diversity in $S$. robustum than in $S$. spontanuem noted in this study. A possible explanation might be the differential capacity among the species to produce tillers and hence biomass. Biomass, other than 
sucrose levels, is another noticeable contrasting trait between the three species. Natural selection for robust plants bearing more tillers led to that species to have a higher capacity for clonal propagation which consequently led to reduced diversity in S. officinarum, and to a lesser extent, in $S$. robustum. Plant biomass yield is highest in $S$. officinarum, then $S$. robustum, and lowest in S. spontaneum.

Based on phylogenetic analysis, the five SuSy genes from sorghum and sugarcane can be classified into the three distinct classes: SuSy1 and SuSy2, SuSy3 and SuSy5, and SuSy4 clustered into Class I, II and III, respectively (Figure 3). Previous studies of SuSy gene family evolution in Arabidopsis, Citrus and Populus showed the existence of three or four distinct SuSy subgroups to exist in plants $[25,32,33]$. Interestingly, compared with the rice SuSy genes, the orthologous OsSuSy3 gene is missing in sorghum. In the same manner that sequence comparison of rice and sorghum revealed about $7 \%$ of the genes appear to be unique to sorghum [9], the OsSuSy3 could be from lineage specific gene duplication event in rice after its divergence from the ancestor of sorghum and sugarcane.

The occurrence of the first SuSy gene duplication event was predicted to be before the angiosperm/ gymnosperm divergence which occurred about 200 mya; and a later duplication of SuSys within subclasses among angiosperms must have arisen before the separation of the monocots and dicots, which is thought to have occurred about 140-150 MYA [34]. The results of the phylogenetic analysis of this study are consistent with the timeline described above. In addition, the predicted molecular weights of the 5 polypeptides are close, ranging from 91.71 to $98.79 \mathrm{kDa}$; and among them, ScSuSy1-4 are around $93 \mathrm{kDa}$, which is consistent with the SDS-PAGE results [21].

The average SNP frequency of ScSuSy genes in the three species is lower than one per 58 bp in the $S$. officinarum, one per $35 \mathrm{bp}$ in the sugarcane hybrid cultivar Q165 [35], and an average of one every 50 bp as occurs by the EST estimation [36]. Based on the SNP frequencies of the Saccarhum species, the predicted SNP frequencies of hybrids between S.officinarum (LA Purple) and S. spontaneum (SES208) is about 1 SNP per 50 bp; this is still higher than the SNP frequencies (one every 35 based ) of sugarcane cultivar Q165. This could be the result of purifying selection in $S c S u S y$, a primary gene family in sucrose metabolism, hence reduce genetic diversity [37].

Since sugarcane is an autopolyploid with each locus having multiple haplotypes from eight or more depending on the ploidy level of the accession. This multiple haplotypes per gene, an indication of heterozygosity level, is likely to have contributed to the high biomass yield of sugarcane. However, there are indications that the increased fixation of elite alleles in modern breeding germplasm is already inhibiting further genetic gain of sugarcane. As modern sugarcane cultivars are derived from crosses between S. officinarum, S. spontaneum, as well as $S$. robustum, analyses of haplotypes and allele complexity of genes in sucrose metabolism in domesticated and wild species will improve our understanding of genetic basis for sucrose accumulation in modern sugarcane cultivars and the level of heterozygosity within the genome of each species.

The haplotype diversity can be seen as an indication of heterozygosity level of both genes and species. All of the $\mathrm{ScSuSy}$ genes, except perhaps $\mathrm{ScSuSy} 5$, showed relatively high levels of heterozygosity (Table 4). It is possible, however, that the short fragment length and random distribution of SNPs, the haplotype number of ScSuSy5 might be only less variable within the length of fragments used for the haplotype analysis. The five $S c S u S y$ family members were evolved before the divergence between sugarcane and sorghum 8 MYA (Figure 1), whereas haplotype diversity in Saccharum occurred after the WGD events less than 1.5 MYA. There is no correlation between ScSuSy family members and haplotype diversity.

SNP frequency does not correlate to haplotype diversity or protein diversity. Among the three species, S. robustum has the most haplotypes (Table 4), not S. spontaneum that has the highest SNP frequency. Moreover, S. officinarum, which has the lowest SNP frequency, has the highest number of deduced protein sequences (Table 5), whereas $S$. spontaneum, which has the highest SNP frequency, has the lowest number of deduced protein sequences. A pairwise $\mathrm{dS} / \mathrm{dN}$ ratio test for selection (Table 5) showed that 10 out of the 19 pairs had $\mathrm{Ka}>=\mathrm{Ks}$; an indication of positive selection. Thus, SNP differences between species could have been the results of positive selection towards accumulation of sucrose in the high sugar content $S$. officinarum and intermediate sugar content in $S$. robustum. Detailed examination of haplotype diversity revealed that the difference of haplotype numbers between the two wild species S. robustum and S. spontaneum is from $S c S u S y 5$ with the maximum of eight haplotypes in $S$. robustum and four haplotypes in S. spontaneum. No transcript from this gene was detected in the five tissue types in all three species. It is not clear whether this gene has a function in sugar metabolism. The analysis of haplotypes provides the opportunity to infer the evolutionary history of a DNA region $[38,39]$. In this study, the consensus haplotypes for the ScSuSy genes in Saccharum species could be used for estimating the origin of haplotypes and discovering the relation among the Saccharum species. The number of consensus haplotypes between $S$. officinarum and $S$. robustum is significantly higher ( $\mathrm{t}$-test, $\mathrm{P}<0.05$ ) than the other two combinations of the three species, which reinforce the notion that the divergence between 
these two species occurred after their common ancestor diverged from S. spontaneum [12,40,41]. A total of 94 haplotypes in 74 unique haplotypes are present in the 1,366 fragments of SuSy genes (Table 4). Of 1,366 fragments, 726 sequences in 17 unique haplotypes are common among the three species. As the three species in the study are octoploid, the haplotypes of the five SuSy genes of species results from the 5 groups of 24 homologous chromosomes. The consensus 17 unique haplotypes, which occur at a frequency of $53.1 \%(726 / 1366)$, are derived from half of the homologous chromosomes. The frequencies of the consensus haplotypes are much higher than any species specific haplotypes, suggesting that the consensus haplotypes were derived from multiple homologous chromosomes. These results reflect the fact that the brief evolutionary history of haplotypes accounts for only a fraction of the time since the divergence of the five $\mathrm{ScSuSy}$ gene members. Selection constraint on these genes in the sucrose biosynthesis and degradation further reduced the diversification of haplotypes.

The SNP frequency within each species and the number of haplotypes within each genome provide crucial information for assessing strategies to sequence these complex genomes. Each homologous chromosome consists of a mosaic of haplotypes sharing various degree of sequence identity with haplotypes in any of the other seven chromosomes. With a SNP frequency at one per 108 bp or higher, it is not possible to have a consensus sequence among eight homologous chromosomes. There is no diploid or tetraploid accessions in Saccharum, and simplest genome is a tetraploid (haploid) accession of S. spontaneum SES 208 generated by anther culture [42]. This genotype would be the best material for sequencing the first genome of Saccharum, and even for that tetraploid genome, ultra long sequence reads from single molecules are needed for correct assembling of the homologous chromosomes and annotation of allelic variations with haplotypes varying from three to eight in homologous regions.

\section{Conclusions}

Analyses of SNP and haplotypes in three primary Saccharum species revealed insights into the level of heterozygosity within each octoploid genome and the evolutionary history of these three genomes. The within genome heterozygosity as measured by SNP frequency is the lowest in the domesticated species S. officinarum and highest in the wild species $S$. spontaneum, suggesting that the WGD events occurred earlier in $S$. spontaneum than in S. officinarum. S. officinarum shared more common SNPs with $S$, robustum than with $S$. spontaneum, confirming the closer phylogenetic relationship between $S$. officinarum and S. robustum. This may also explain the success of integrating disease/pest resistance genes from $S$. spontaneum as these two species contain more diverse sets of $\mathrm{R}$ genes than between
S. officinarum and S. robustum. Although the number of haplotypes is fewer in $S$. officinarum than in $S$. spontaneum, the number of deduced protein sequences is higher in $S$. officinarum than in S. spontaneum, a sign of positive selection on these ScSuSy genes in the high sugar content species $S$. officinarum.

\section{Methods}

\section{Plant materials}

Three varieties of Saccharum species were used in the study: $S$. officinarum LA Purple $(2 \mathrm{n}=8 \times=80), \quad S$. robustum Molokai $6081(2 \mathrm{n}=8 \times=80)$, and S. spontaneum SES208 $(2 n=8 \times=64)$ [3]. Genomic DNA from young leaf tissues for each of the three accessions were isolated using Qiagen DNeasy miniprep kit following the manufacturer's protocol (Qiagen, Inc., Valencia, CA, USA).

\section{Database Searches and gene Annotation for the SuSy genes in sorghum}

Six Arabidopsis SuSy sequences (At1G73370, AT1G73370, AT5G2083, At5g49190, At5g37180, and At4g02280) and six rice SuSy sequences [24], to identify the full set of SuSy genes in the sorghum (Sorghum bicolor) genome. BLASTn and tBLASTn search (http://blast.ncbi.nlm.nih.gov/) hits that has similarity scores of $>50.0$ and probability scores of $<10^{-4}$ were retained for further analysis. Wherever possible, we checked the published annotations of the sorghum genomic clones against full-length cDNA clones and ESTs from sugarcane and sorghum in NCBI. We also checked the predicted amino acid sequences against the conserved motifs of SuSy. For genomic sequences that had not previously been annotated, we supplemented the above methods with the use of Genscan (http://genes.mit. edu/GENSCAN.html) [43] and FGENESH (http://linux1. softberry.com/berry.phtml) gene prediction software. SuSy protein sequences were analyzed using tools available at http://us.expasy.org/tools/.

\section{Verification of SuSy genes members in sugarcane}

The genomic and predicted mRNA sequences of sorghum were used to search the ESTs database of sugarcane. Based on the sorghum SuSy genes, the ESTs hits of sugarcane were classified for predicting the number the SuSy genes in sugarcane. PCR primers were designed, using Primer Premier c5.00, from the sorghum genome and sugarcane EST sequences to amplify an approximately 500nt, introncontaining region in the Saccharum genome (Additional file 7). PCR reactions were carried out in a total of $50 \mu \mathrm{l}$ volume containing $30 \mathrm{ng}$ template DNA, $0.2 \mu \mathrm{M}$ of each PCR primer and $25 \mu \mathrm{l} 2 \times \mathrm{GoTaq}^{\circledR}$ Green Master Mix (Promega, WI, USA). PCR conditions were: $3 \mathrm{~min}$ at $94^{\circ} \mathrm{C}$ followed by 35 cycles of $10 \mathrm{~s}$ at $94^{\circ} \mathrm{C}, 30 \mathrm{~s}$ at the appropriate annealing temperature $\left(50-65^{\circ} \mathrm{C}\right), 30 \mathrm{~s}$ at $72^{\circ} \mathrm{C}$ and an 
additional extension step of $6 \mathrm{~min}$ at $72^{\circ} \mathrm{C}$. PCR amplification was verified by running samples out on a $1 \%$ agarose gel.

The primers for genomic amplification were also used for verifying $S u S y$ transcripts by RT-PCR (Additional file 7). Using TRIzol ${ }^{\circledR}$ (Invitrogen, USA), total RNA was extracted from two different stem, mature leaf and leaf roll of three Saccharum species. The total RNA was treated with RNase-free DNase I(Ambion, TX,USA) and reverse transcribed using ImProm- $\mathrm{II}^{\mathrm{TM}}$ Reverse Transcription System (Promega, WI, USA). RT-PCR reactions were carried out as previously described.

In addition, using the predicted sorghum SuSy cDNA sequence as a reference, a cDNA database from Illumina RNA-seq sequencing with 42 million pair-end reads were searched by NOVOALIGN with default parameters (http://www.novocraft.com/main/index.php). The sequences of target genes from NOVOALIGN results were obtained using Tablet software [44] and assembled by Sequencher 4.0 (Minimum Match Percentage 96\%, Minimum overlap 20\%) to achieve the full cDNA sequences of the SuSy genes. The corresponding amino acid sequences of the SuSy genes were deduced through BLASTx and ORF finder (http://www.ncbi.nlm.nih.gov/gorf/gorf.html).

\section{Phylogenetic dendrogram of SuSy gene family members}

The amino acid sequences of SuSy gene family members including members from angiosperms, gymnosperms and bacteria, were identified by searching public databases available at NCBI (http://www.ncbi.nlm.nih.gov). A phylogenetic dendrogram of SuSy members was made using the deduced amino acid sequences by ClustalW v2 program (http://www.ebi.ac.uk/Tools/msa/clustalw2/). The tree view program version 1.6.6 [45] was used to generate unrooted trees with the stability of the tree obtained estimated by bootstrap analysis for 100 replications.

\section{Identification of SNPs and Haplotype Analysis of the scSuSy Gene in Saccarhum species}

We cloned the gene fragments following the modified procedure done to characterize the SPS III in sugarcane [29]. Each DNA samples from three Saccharum species was amplified twice for the SuSy genes. The resulting amplified PCR products were then purified using Wizard $^{\circledR}$ SV Gel and PCR Clean-Up System (Promega, WI, USA) cloned into pGEMT Easy Vector (Promega, WI, USA) and transformed into chemically competent E. coli strain JM109. Individual colonies were grown in LB media at $37 \mathrm{C}$ overnight, and plasmids were isolated using a modified alkaline lysis procedure. The purified fragments were sequenced using the $\mathrm{T} 7$ primer and the big dye terminator cycle sequencing kit (Applied Biosystems) performed by the W.M. Keck Center for Comparative and Functional Genomics, University of
Illinois at Urbana-Champaign ( http://www.biotech.uiuc. $\mathrm{edu} /$ ). To avoid confounding error from PCR recombination and sequencing, only SNPs and haplotypes that were observed in at least two colonies were considered for sequencing.

The generated sequence reads were inspected and trimmed manually for quality using Sequencher 4.10.1. The sequence reads of each of the sbSuSy gene fragment for each saccharum species were separately aligned to identify the sbSuSy SNPs and SNPs haplotypes within species. Conversely, all the SuSy gene sequences were aligned together to investigate SNP polymorphism between the three saccharum species.

The unique combinations of SNPs found after alignment were used to define haplotypes for each scSusy gene per species. Assessment of haplotype SNP counts and SNP frequency were assessed manually. From these alignments as well, the protein sequences were predicted by BlastX and aligned with ClustalW [46], converted back to DNA (codon) alignments with PAL2NAL [47] from which synonymous substitutions (dS) and nonsynonymous substitutions $(\mathrm{dN})$ was calculated following the same pipeline as described in an earlier report [14].

\section{Additional files}

Additional file 1: List of sucrose synthase gene sequences used in this study.

Additional file 2: The SNPs frequencies in the 5 SuSy genes of Three Saccharum Species.

Additional file 3: SNP position within the SuSy haplotype fragment of the Saccharum species.

Additional file 4: Summary of the estimated haplotype analysis of SuSy genes in three Saccharum species.

Additional file 5: The predicted amino acid of the haplotypes of SuSy genes fragments from the Saccharum species.

Additional file 6: Summary of Ka, Ks calculation.

Additional file 7: The primers for genes verification and haplotype analysis.

\section{Competing interests}

The authors declare that they have no competing interests.

\section{Authors' contributions}

$J Z$ and RM conceived the study and designed the experiments. JZ carried out the experiments and analyzed the data. JZ and RM wrote the manuscript. JA and YC analyzed the data and contributed to the writing of the manuscript. All authors read and approved the final paper.

\section{Acknowledgements}

We are grateful for the invaluable help and insights of Dr. Haibao Tang, especially in the data analyses section such as Ka/Ks analysis. This project was supported by the International Consortium for Sugarcane Biotechnology, the Consortium for Plant Biotechnology Research, the National Natural Science Foundation of China (31201260) and 863 program (2013AA100604).

\section{Author details}

${ }^{1}$ College of Life Sciences, Fujian Normal University, Fuzhou 350108, China. ${ }^{2}$ Department of Plant Biology, University of Illinois at Urbana-Champaign, Urbana, IL 61801, USA. 
Received: 30 October 2012 Accepted: 29 April 2013

Published: 10 May 2013

\section{References}

1. Lam E, Shine J, Da Silva J, Lawton M, Bonos S, Calvino M, Carrer H, Silva-Filho MC, Glynn N, Helsel Z, et al: Improving sugarcane for biofuel: engineering for an even better feedstock. Gcb Bioenergy 2009, 1(3):251-255.

2. Irvine JE: Saccharum species as horticultural classes. Theor Appl Genet 1999, 98(2):186-194.

3. Zhang JS, Nagai C, Yu QY, Pan YB, Ayala-Silva T, Schnell RJ, Comstock JC, Arumuganathan AK, Ming R: Genome size variation in three Saccharum species. Euphytica 2012, 185(3):511-519

4. Cuadrado A, Acevedo R, de la Espina SMD, Jouve N, de la Torre C: Genome remodelling in three modern S-officinarumxS-spontaneum sugarcane cultivars. J Exp Bot 2004, 55(398):847-854.

5. D'Hont A, Grivet L, Feldmann P, Rao S, Berding N, Glaszmann JC Characterisation of the double genome structure of modern sugarcane cultivars (Saccharum spp.) by molecular cytogenetics. Mol Gen Genet 1996, 250(4):405-413.

6. Piperidis $\mathrm{G}$, $\mathrm{D}^{\prime}$ Hont $\mathrm{A}$ : Chromosome composition analysis of various Saccharum interspecific hybrids by genomic in situ hybridisation (GISH), Proceedings of the XXIV ISSCT Congress: September 17-21 2001. Brisbane, Australia: International Society of Sugar Cane Technologists; 2001.

7. Borecky J, Nogueira FTS, de Oliveira KAP, Maia IG, Vercesi AE, Arruda P: The plant energy-dissipating mitochondrial systems: depicting the genomic structure and the expression profiles of the gene families of uncoupling protein and alternative oxidase in monocots and dicots. J Exp Bot 2006, 57(4):849-864

8. Sculaccio SA, Napolitano HB, Beltramini LM, Oliva G, Carrilho E, Thiemann $\mathrm{OH}$ : Sugarcane Phosphoribosyl Pyrophosphate Synthetase: Molecular Characterization of a Phosphate-independent PRS. Plant Mol Biol Rep 2008, 26(4):301-315.

9. Paterson AH, Bowers JE, Bruggmann R, Dubchak I, Grimwood J, Gundlach H, Haberer G, Hellsten U, Mitros T, Poliakov A, et al: The Sorghum bicolor genome and the diversification of grasses. Nature 2009, 457(7229):551-556.

10. Dufour P, Deu M, Grivet L, DHont A, Paulet F, Bouet A, Lanaud C, Glaszmann JC, Hamon P: Construction of a composite sorghum genome map and comparison with sugarcane, a related complex polyploid. Theor Appl Genet 1997, 94(3-4):409-418.

11. Guimaraes $C T$, Sills GR, Sobral BWS: Comparative mapping of Andropogoneae: Saccharum L. (sugarcane) and its relation to sorghum and maize. P Natl Acad Sci USA 1997, 94(26):14261-14266.

12. Ming R, Liu SC, Lin YR, da Silva J, Wilson W, Braga D, van Deynze A, Wenslaff TF, Wu KK, Moore PH, et al: Detailed alignment of Saccharum and Sorghum chromosomes: Comparative organization of closely related diploid and polyploid genomes. Genetics 1998, 150(4):1663-1682.

13. Jannoo N, Grivet L, Chantret N, Garsmeur O, Glaszmann JC, Arruda P, D'Hont A: Orthologous comparison in a gene-rich region among grasses reveals stability in the sugarcane polyploid genome. Plant J 2007, 50(4):574-585.

14. Wang J, Roe B, Macmil S, Yu Q, Murray JE, Tang H, Chen C, Najar F, Wiley G, Bowers J, et al: Microcollinearity between autopolyploid sugarcane and diploid sorghum genomes. BMC Genomics 2010, 11:261.

15. Chourey PS, Taliercio EW, Carlson SJ, Ruan YL: Genetic evidence that the two isozymes of sucrose synthase present in developing maize endosperm are critical, one for cell wall integrity and the other for starch biosynthesis. Mol Gen Genet 1998, 259(1):88-96.

16. Koch $\mathrm{KE}, \mathrm{Wu} Y, \mathrm{Xu}$ J: Sugar and metabolic regulation of genes for sucrose metabolism: potential influence of maize sucrose synthase and soluble invertase responses on carbon partitioning and sugar sensing. J Exp Bot 1996, 47:1179-1185.

17. N'tchobo H, Dali N, Nguyen-Quoc B, Foyer CH, Yelle S: Starch synthesis in tomato remains constant throughout fruit development and is dependent on sucrose supply and sucrose synthase activity. J Exp Bot 1999, 50(338):1457-1463.

18. Schrader S, Sauter JJ: Seasonal changes of sucrose-phosphate synthase and sucrose synthase activities in poplar wood (Populus $x$ canadensis Moench < robusta $>$ ) and their possible role in carbohydrate metabolism. J Plant Physiol 2002, 159(8):833-843.

19. Lingle SE: Sugar metabolism during growth and development in sugarcane internodes. Crop Sci 1999, 39(2):480-486.
20. Botha FC, Black KG: Sucrose phosphate synthase and sucrose synthase activity during maturation of internodal tissue in sugarcane. Aust J Plant Physiol 2000, 27(1):81-85

21. Lingle SE, Dyer JM: Cloning and expression of sucrose synthase-1 CDNA from sugarcane. J Plant Physiol 2001, 158(1):129-131.

22. Carlson SJ, Chourey PS, Helentjaris T, Datta R: Gene expression studies on developing kernels of maize sucrose synthase (SuSy) mutants show evidence for a third SuSy gene. Plant Mol Biol 2002, 49(1):15-29.

23. Duncan KA, Hardin SC, Huber SC: The three maize sucrose synthase Isoforms differ in distribution, localization, and phosphorylation. Plant Cell Physiol 2006, 47(7):959-971.

24. Hirose T, Scofield GN, Terao T: An expression analysis profile for the entire sucrose synthase gene family in rice. Plant Sci 2008, 174(5):534-543.

25. Bieniawska Z, Barratt DHP, Garlick AP, Thole V, Kruger NJ, Martin C, Zrenner R, Smith AM: Analysis of the sucrose synthase gene family in Arabidopsis. Plant J 2007, 49(5):810-828.

26. Zhang X, Zong J, Liu JH, Yin JY, Zhang DB: Genome-Wide Analysis of WOX Gene Family in Rice, Sorghum, Maize, Arabidopsis and Poplar. J Integr Plant Biol 2010, 52(11):1016-1026.

27. Barrero-Sicilia C, Hernando-Amado S, Gonzalez-Melendi P, Carbonero P. Structure, expression profile and subcellular localisation of four different sucrose synthase genes from barley. Planta 2011, 234(2):391-403.

28. Carson DL, Botha FC: Genes expressed in sugarcane maturing internodal tissue. Plant Cell Rep 2002, 20(11):1075-1081.

29. McIntyre CL, Jackson M, Cordeiro GM, Amouyal O, Hermann S, Aitken KS, Eliott F, Henry RJ, Casu RE, Bonnett GD: The identification and characterisation of alleles of sucrose phosphate synthase gene family III in sugarcane. Mol Breeding 2006, 18(1):39-50.

30. Garsmeur O, Charron C, Bocs S, Jouffe V, Samain S, Couloux A, Droc G, Zini C, Glaszmann JC, Van Sluys MA, et al: High homologous gene conservation despite extreme autopolyploid redundancy in sugarcane. New Phytol 2011, 189(2):629-642.

31. Yang ZH: PAML 4: Phylogenetic analysis by maximum likelihood. Mol Biol Evol 2007, 24(8):1586-1591.

32. Zhang PF, Dreher K, Karthikeyan A, Chi A, Pujar A, Caspi R, Karp P, Kirkup V Latendresse M, Lee $C$, et al: Creation of a Genome-Wide Metabolic Pathway Database for Populus trichocarpa Using a New Approach for Reconstruction and Curation of Metabolic Pathways for Plants. Plant Physiol 2010, 153(4):1479-1491.

33. Komatsu A, Moriguchi T, Koyama K, Omura M, Akihama T: Analysis of sucrose synthase genes in citrus suggests different roles and phylogenetic relationships. J Exp Bot 2002, 53(366):61-71.

34. Chaw SM, Chang CC, Chen HL, Li WH: Dating the monocot-dicot divergence and the origin of core eudicots using whole chloroplast genomes. J Mol Evol 2004, 58(4):424-441.

35. Bundock PC, Eliott FG, Ablett G, Benson AD, Casu RE, Aitken KS, Henry RJ: Targeted single nucleotide polymorphism (SNP) discovery in a highly polyploid plant species using 454 sequencing. Plant Biotechnol J 2009, 7(4):347-354.

36. Cordeiro GM, Eliott F, McIntyre CL, Casu RE, Henry RJ: Characterisation of single nucleotide polymorphisms in sugarcane ESTs. Theor Appl Genet 2006, 113(2):331-343.

37. Maruki T, Kumar S, Kim Y: Purifying Selection Modulates the Estimates of Population Differentiation and Confounds Genome-Wide Comparisons across Single-Nucleotide Polymorphisms. Mol Biol Evol 2012, 29(12):3617-3623.

38. Templeton AR, Sing CF, Kessling A, Humphries S: A Cladistic-Analysis of Phenotype Associations with Haplotypes Inferred from Restriction Endonuclease Mapping.2. The Analysis of Natural-Populations. Genetics 1988, 120(4):1145-1154.

39. Tishkoff DX, Amin NS, Viars CS, Arden KC, Kolodner RD: Identification of a human gene encoding a homologue of Saccharomyces cerevisiae EXO1, an exonuclease implicated in mismatch repair and recombination. Cancer Res 1998, 58(22):5027-5031.

40. Brown JS, Schnell RJ, Power EJ, Douglas SL, Kuhn DN: Analysis of clonal germplasm from five Saccharum species: S. barberi, $\mathrm{S}$. robustum, $\mathrm{S}$. officinarum, S. sinense and S. spontaneum. A study of inter- and intra species relationships using microsatellite markers. Genet Resour Crop Ev 2007, 54(3):627-648

41. Daniels J, Roach B: Taxonomy and Evolution. In Sugarcane Improvement through Breeding. Edited by Heinz D. Amsterdam: Elsevier Press; 1987:7-84. 
42. Moore PH, Nagai C, Fitch M: Production and Evaluation of Sugarcane Hybrids, Proceedings of International Soceity of Sugarcane Technologists XX Congress: 1989. Sao Paulo: ISSCT; 1989:599-601.

43. Burge C, Karlin S: Prediction of complete gene structures in human genomic DNA. J Mol Biol 1997, 268(1):78-94.

44. Milne I, Bayer M, Cardle L, Shaw P, Stephen G, Wright F, Marshall D: Tabletnext generation sequence assembly visualization. Bioinformatics 2010, 26(3):401-402.

45. Page RDM: TreeView: An application to display phylogenetic trees on personal computers. Comput Appl Biosci 1996, 12(4):357-358.

46. Larkin MA, Blackshields G, Brown NP, Chenna R, McGettigan PA, McWilliam $H$, Valentin F, Wallace IM, Wilm A, Lopez R, et al: Clustal W and Clustal X version 2.0. Bioinformatics 2007, 23(21):2947-2948.

47. Suyama M, Torrents D, Bork P: PAL2NAL: robust conversion of protein sequence alignments into the corresponding codon alignments. Nucleic Acids Res 2006, 34:W609-W612.

doi:10.1186/1471-2164-14-314

Cite this article as: Zhang et al:: Haplotype analysis of sucrose synthase gene family in three Saccharum species. BMC Genomics 2013 14:314.

\section{Submit your next manuscript to BioMed Central and take full advantage of:}

- Convenient online submission

- Thorough peer review

- No space constraints or color figure charges

- Immediate publication on acceptance

- Inclusion in PubMed, CAS, Scopus and Google Scholar

- Research which is freely available for redistribution 